\title{
Renal Abscess with Morganella morganii Complicating Leukemoid Reaction
}

\author{
Shinobu Osanai, Hiroaki Nakata, Kensuke Ishida, Mie Hiramatsu, Eri Toyoshima, \\ Toshiyuki Ogasa, Yoshinobu Ohsaki and Kenjiro Kikuchi
}

\begin{abstract}
We report a case of leukemoid reaction (LR) complicating renal abscess caused by Morganella morganii infection in an 80-year-old man. On administration, laboratory tests revealed white blood cell count of $76160 / \mu \mathrm{L}$ and $\mathrm{C}$ reactive protein $3.09 \mathrm{mg} / \mathrm{dL}$. Although chronic myeloid leukemia was suspected, bcr/abl fusion transcript was not observed. Contrast enhanced computer tomography imaging of the abdomen showed abscess in the right kidney. M. morganii was detected repeatedly in material of liquid from the abscess and arterial blood culture. To our knowledge, this is the first case of M. morganii infection complicating LR.
\end{abstract}

Key words: bacteremia, renal abscess, Morganella morganii, leukemoid reaction

(DOI: 10.2169/internalmedicine.47.0343)

\section{Introduction}

A leukemoid reaction (LR) is a hematological abnormality, defined by persistent neutrophilia of 25,000 to 30,000 cells/ $\mu \mathrm{L}$ or greater, and induced by reactive causes outside the bone marrow (1). The major causes of LRs are severe infections, intoxications, malignancies, severe hemorrhage, or acute hemolysis (2). A variety of infections, such as Clostridium difficile colitis (3), disseminated tuberculosis (4), and severe shigellosis (5) have been associated with the LR. To date, however, there is no report of the LR induced by Morganella morganii infection.

\section{Case Report}

An 80-year-old man was transferred to the emergency department from a regional hospital because of a high fever and significantly increased white blood cells. One week before admission of the regional hospital, he began to have a high fever with chills, but his history did not suggest any infectious pathology of the biliary, urinary, or respiratory tracts. In past medical history, he had undergone a radical cystourethrectomy with ileal conduit due to bladder cancer eight years previously. A nephrostomy catheter had been inserted in his right kidney and exchanged monthly. He had been occasionally affected by urinary tract infection and had undergone administration of oral cephalosporins or fluoroquinolones.

On the day of admission, he appeared ill and his vital signs included a high fever $\left(41.2^{\circ} \mathrm{C}\right)$, heart rate 108 beats/ minute, blood pressure $96 / 66 \mathrm{mmHg}$ and respiratory rate 22 breaths/minute. Physical examination revealed diffuse, bilateral rhonchi. There was neither heart murmur, peripheral edema, digital clubbing, nor lymphadenopathy. Although there was neither tenderness on abdomen nor ascites, he had mild splenomegaly.

Urinalysis showed small amounts of red blood cells, white blood cells and bacteria. Hematological tests revealed significant leucocytosis, mild anemia and thrombopenia (Table 1). Analysis of arterial blood showed that the partial pressure of oxygen was $53 \mathrm{mmHg}$, the partial pressure of carbon dioxide was $36 \mathrm{mmHg}$, and the $\mathrm{pH}$ was 7.48. An electrocardiogram revealed sinus tachycardia at a rate of 130. A radiograph of the chest (Fig. 1) showed bilateral diffuse infiltration. Microscopical examination of a specimen of sputum showed microbes of normal flora and a few neutrophils.

Myeloproliferative diseases including chronic myeloid leukemia (CML) and chronic neutrophilic leukemia (CNL) were suspected because of marked leucocytosis and splenomegaly. Bone marrow aspiration from the sternum 
Table 1. Hematological and Blood Chemical Values on Admission

\begin{tabular}{|c|c|}
\hline VARIABLE & VALUE \\
\hline$\overline{\text { Red cell count (cells/ } \mu \mathrm{L})}$ & $303 \times 10^{4}$ \\
\hline Hemoglobin $(\mathrm{g} / \mathrm{dL})$ & 7.9 \\
\hline Hematocrit $(\%)$ & 25.6 \\
\hline White cell count (cells/ $\mu \mathrm{L})$ & 76160 \\
\hline \multicolumn{2}{|l|}{ Differential count $(\%)$} \\
\hline Neutrophils & 16 \\
\hline Band forms & 5 \\
\hline Metamyelocytes & 13 \\
\hline Myelocytes & 43 \\
\hline Promyelocytes & 3 \\
\hline Lymphocytes & 7 \\
\hline Monocytes & 13 \\
\hline Eosinophils & 0 \\
\hline Platelet count (cells/ $\mu \mathrm{L})$ & $7.8 \times 10^{4}$ \\
\hline Protein, total $(\mathrm{g} / \mathrm{dL})$ & 5.7 \\
\hline $\operatorname{Albumin}(\mathrm{g} / \mathrm{dL})$ & 2.9 \\
\hline Bilirubin, total (mg/dL) & 1.6 \\
\hline Aspartate aminotransferase (U/L) & 63 \\
\hline Lactate dehydrogenase $(\mathrm{U} / \mathrm{L})$ & 1106 \\
\hline Alkaline phosphatase (U/L) & 264 \\
\hline Urea nitrogen $(\mathrm{mg} / \mathrm{dL})$ & 37 \\
\hline Creatinine (mg/dL) & 0.84 \\
\hline Sodium $(\mathrm{mmol} / \mathrm{L})$ & 146 \\
\hline Potassium (mmol/L) & 3.7 \\
\hline Chloride (mmol/L) & 103 \\
\hline Calcium (mg/dL) & 8.0 \\
\hline Phosphorus (mg/dl) & 4.1 \\
\hline $\mathrm{C}$ reactive protein $(\mathrm{mg} / \mathrm{dL})$ & 3.09 \\
\hline
\end{tabular}
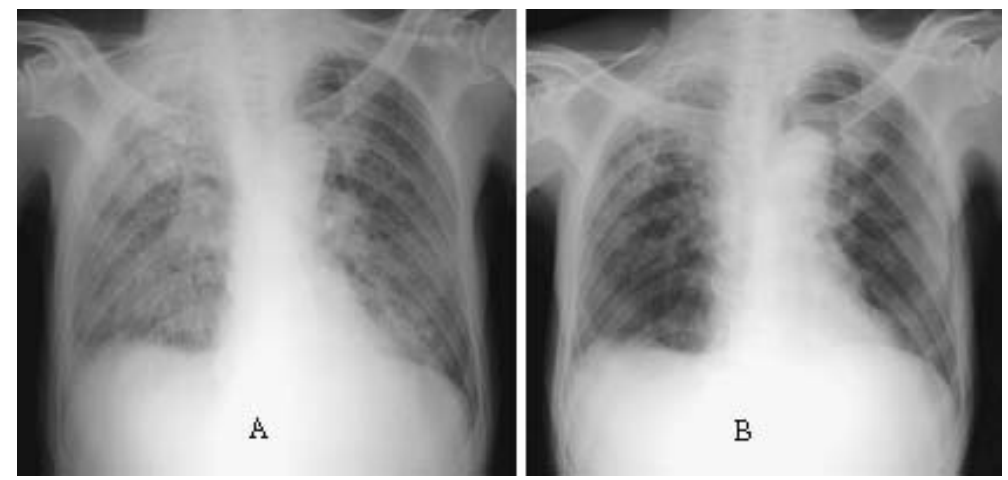

Figure 1. A: Chest X-ray on admission. B: Chest X-ray after treatment with antibiotics.

showed myeloid hyperplasia without abnormal morphological appearance. Neither excess of blast nor abnormal cells was found. Although cytogenetic study was not undertaken, bcr/abl fusion transcript was not observed by fluorescence in situ hybridization. Therefore, CML was denied as being the possible cause of the marked leucocytosis. The differential diagnosis between LR and CNL is very complicated because both conditions share identical morphological features, including an elevated LAP score and the absence of the bcr/ abl translocation $(6,7)$. In the present case, we diagnosed the significant leucocytosis as LR rather than CNL because uncontrolled infection emerged (7). At that point, we diagnosed his illness as LR complicating pneumonia and treated him with antibiotics.

His clinical course in shown in Fig. 2. By administration of ampicillin/sulbactam ( $3 \mathrm{~g}$ i.v., 12 hourly) for seven days and methylprednisolone (500 $\mathrm{mg} /$ day) for three days, his pneumonia was improved. However, his low grade fever and marked leucocytosis continued. Enhanced abdominal CT suggested an abscess in the corticomedullary junction of the 


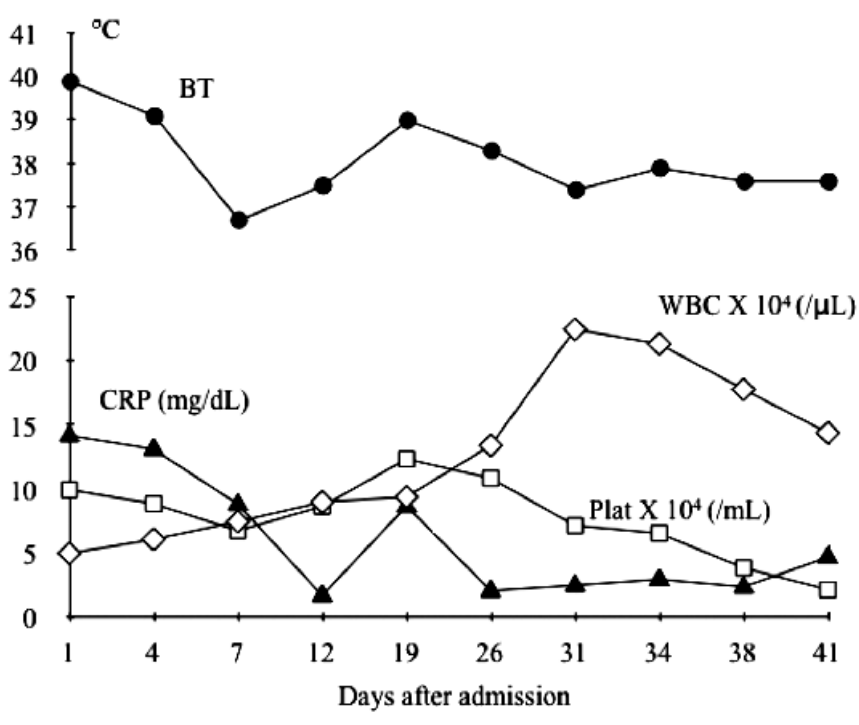

Figure 2. Clinical course. BT: Body temperature, WBC: white blood cell, CRP: $C$ reactive protein, Plat: Platelet

right kidney (Fig. 3). On the other hand, there was no finding that revealed the recurrence of bladder cancer or other neoplasms. Due to poor his general condition a major operation was not recommended and the patient underwent echoguided percutaneous drainage of the renal abscess. We started administration of broad-spectrum antibiotic drug (biapenem 0.3 g i.v., 8 hourly) as an empiric therapy (8). Then, M. morganii was detected repeatedly in material of liquid from abscess and arterial blood culture. Antimicrobial susceptibility tests showed resistance to amoxicillin, minocycline and fosfomycin (Table 2). Carbapenem and third generation cephalosporins were sensitive to $M$. morganii from both the renal abscess and arterial blood culture. After administration of biapenem, the general condition and laboratory findings were not improved. Therefore, we switched the antibiotics to ceftazidim ( $1 \mathrm{~g}$ i.v., 8 hourly) referring to published results on the treatment of $M$. morganii (9). In spite of high dosage of antibiotics and other supportive treatments, sepsis was progressive and developed into disseminated intravascular coagulation. He died after 2 weeks of percutaneous puncture.

\section{Discussion}

LR can follow exposure to drugs, such as corticosteroids, minocycline, recombinant hematopoietic growth factors, or to toxin such as ethylene glycol (2). Since we used high dose of methylprednisolone in this case after marked leucocytosis, it was impossible to relate corticosteroids and LR. After administration of corticosteroids, its effect might only modify leucocytosis in the clinical course. In addition, other drugs which are reported to cause of LR were not administrated before the onset of LR.

Other disorders that can cause LR are mesenteric inflammatory pseudotumor, alcoholic steatohepatitis, and retroperi-

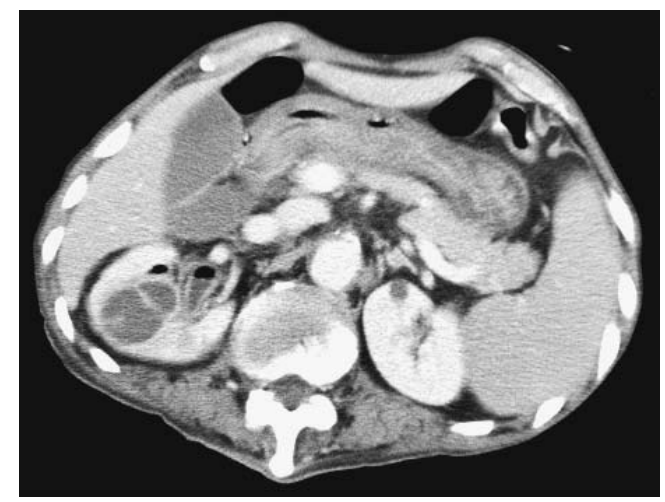

Figure 3. Contrast-enhanced computer tomography imaging of the abdomen shows a focal area of decreased attenuation within the right renal parenchyma, with the involved portion appearing swollen. In the involved area, there was also a round, low attenuation structure with rim enhancement. There was small amount of air in the pelvis of right kidney.

toneal hemorrhage (2). In addition, LRs can present simultaneously with the malignancy. Malignancy-associated LRs are commonly observed in a variety of carcinomas, most notably lung and kidney cancer (2). In the present case, those causes of LRs were denied from clinical history and findings.

Renal abscess can arise from both an initial urinary tract infection (UTI) and hematogenous spreading of bacteria from a primary focus of infection outside the kidney (10). Generally, renal abscess due to UTI is often coupled with urinary tract abnormalities including vesicoureteral reflex, nephrolithiasis, urinary tract obstruction $(10,11)$. In those cases, enteric gram-negative bacteria are the typical infecting organisms $(10,11)$. In the present case, the patient had ileal conduit and the nephrostomy catheter in the right kidney, and he had occasionally been affected by urinary tract infection.

In addition, there was no primary focus of $M$. morganii infection in this case. Therefore, we presumed that the present case belonged to the high risk group of renal abscess which developed as an ascending infection.

People requiring long-term urinary tract draining commonly experience catheter-associated UTI (CAUTI) and other problems. Previously, numerous trials have evaluated methods to decrease the risk of CAUTI. Some trials showed improvements in management of the catheters can reduce the risk of CAUTI (12). Also, antibiotic drug prophylaxis for CAUTI is valuable in patients with surgical treatments. Although these methods can prevent CAUTI for weeks at most, there is no effective precaution against CAUTI in long-term catheterized patients like the present case (12).

M. morganii is a gram-negative rod commonly found in the environment and in the intestinal tracts of humans as normal flora (13). Although $M$. morganii ranges widely in the environment, it is an uncommon cause of communityacquired infection. It is most often encountered in postop- 
DOI: $10.2169 /$ internalmedicine.47.0343

Table 2. Resistance Profile of Morganella morganii Isolated from Arterial Blood and Renal Abscess

\begin{tabular}{lllll}
\hline Antibiotics & \multicolumn{2}{l}{ Material } & & \\
& \multicolumn{2}{l}{ arterial blood } & \multicolumn{2}{c}{ renal abscess } \\
& MIC & Inter. & MIC & Inter. \\
\hline Amoxicillin & $64 \leq$ & $\mathrm{R}$ & $64 \leq$ & $\mathrm{R}$ \\
Piperacillin & $\leq 0.5$ & $\mathrm{~S}$ & $\leq 0.5$ & $\mathrm{~S}$ \\
Cefotiam & 8 & $\mathrm{~S}$ & 8 & $\mathrm{~S}$ \\
Cefotaxime & $\leq 0.5$ & $\mathrm{~S}$ & $\leq 0.5$ & $\mathrm{~S}$ \\
Ceftazidime & $\leq 0.5$ & $\mathrm{~S}$ & $\leq 0.5$ & $\mathrm{~S}$ \\
Cefozopran & $\leq 0.5$ & $\mathrm{~S}$ & $\leq 0.5$ & $\mathrm{~S}$ \\
Cefoperazone-sulbactam & 1 & $\mathrm{~S}$ & 1 & $\mathrm{~S}$ \\
Flomoxef & $\leq 0.5$ & $\mathrm{~S}$ & $\leq 0.5$ & $\mathrm{~S}$ \\
Imipenem-cilastatin & 1 & $\mathrm{~S}$ & 1 & $\mathrm{~S}$ \\
Amikacin & $\leq 0.5$ & $\mathrm{~S}$ & $\leq 0.5$ & $\mathrm{~S}$ \\
Gentamicin & $\leq 0.5$ & $\mathrm{~S}$ & $\leq 0.5$ & $\mathrm{~S}$ \\
Minocycline & 16 & $\mathrm{R}$ & 16 & $\mathrm{R}$ \\
Levofloxacin & $\leq 0.25$ & $\mathrm{~S}$ & $\leq 0.25$ & $\mathrm{~S}$ \\
Fosfomycin & 64 & $\mathrm{R}$ & 64 & $\mathrm{R}$ \\
Sulfamethoxazole & $\leq 4.75$ & $\mathrm{~S}$ & $\leq 4.75$ & $\mathrm{~S}$ \\
-trimethoprim & & & & \\
\hline & & & & \\
\hline
\end{tabular}

Susceptibilities were determined by microdilution reference method. Interpretive criteria are per National Committee for Clinical Laboratory Standards Guideline. MIC, minimal inhibitory concentration $(\mu \mathrm{g} / \mathrm{ml})$; Inter., interpretation; S, sensitive; R, resistant.

erative or other nosocomial situations $(14,15)$. The most frequent cause of $M$. morganii bacteremia is postoperative wound infection, and most infections occur in patients who have received recent therapy with beta-lactam antibiotics (15). Other important epidemiological risk factors in these studies was the presence of diabetes mellitus or other serious underlying diseases. Several factors were applicable to developed $M$. morganii bacteremia in the present case.

It has been reported that various infections induce the LRs (15). The causes of LRs in infections, however, are not fully understood. In innate immunity, some components from microbes are recognized in receptors on host cells. For example, lipopolysaccharid (LPS), or endotoxin, from gramnegative bacterial cell wall can stimulate macrophages. In the macrophage responses to LPS, LPS-binding protein, CD 14 and Toll-like receptors mainly mediate (16). Stimulated macrophage will produce various humoral factors including tumor necrosis factor (TNF), granulocyte colony-stimulating factor (G-CSF), granulocyte-macrophage colony-stimulating factor (GM-CSF), interleukin-1 (IL-1) and so on (17). It has been implicated that these humoral factors can increase leucocytes in the peripheral blood with moving from storage pool and stimulating production in bone marrow. There is LPS in the outer membrane of M. morganii, as in other gram negative bacteria (18). Under the condition of uncontrolled M. morganii bacteremia, it is likely that an excessive amount of LPS or other components from microbes can stimulate the immune system and increase the neutrophils in peripheral blood.

To our knowledge, this is the first case of renal abscess with $M$. morganii complicating LR. However, similar cases will be encountered more frequently in the future because of the increase of population with high risks in Japan.

\section{References}

1. Bagby GC Jr. Leukopenia and leukocytosis. In: Textbook of Medicine. 21st ed. Goldman L, Bennett JC, Eds. W.B. Saunders, Philadelphia, 2000: 919-933.

2. Sakka V, Tsiodras S, Giamarellos-Bourboulis EJ, Giamarellou H. An update on the etiology and diagnostic evaluation of a leu- kemoid reaction. Eur J Intern Med 17: 394-398, 2006.

3. Marinella MA, Burdette SD, Bedimo R, Markert RJ. Leukemoid reactions complicating colitis due to Clostridium difficile. South Med J 97: 959-963, 2004.

4. Au WY, Ma SK, Kwong YL. Disseminated hepatosplenic myco- 
bacterial infection masking myeloproliferative diseases as leukemoid reaction: a diagnostic pitfall. Leuk Lymphoma 42: 805808, 2001.

5. Azim T, Qadri F, Ahmed S, et al. Lipopolysaccharide-specific antibodies in plasma and stools of children with Shigella-associated leukemoid reaction and hemolytic-uremic syndrome. Clin Diagn Lab Immunol 3: 701-705, 1996.

6. Bohm J, Kock S, Schaefer HE, Fisch P. Evidence of clonality in chronic neutrophilic leukaemia. J Clin Pathol 56: 292-295, 2003.

7. Imbert M, Vardiman JW, Bain B, et al. Chronic myeloproliferative diseases. In: World Health Organization Classification of Tumours. Pathology and Genetics of Tumours of Haematopoietic and Lymphoid Tissues. Jaffe ES, Harris NL, Stein H, Vardiman JW, Eds. IARC Press, Lyon, 2001: 20-28.

8. Clarke AM, Zemcov SJ. Comparative in vitro activity of biapenem, a new carbapenem antibiotic. Eur J Clin Microbiol Infect Dis 12: 377-384, 1993.

9. Lee IK, Liu JW. Clinical characteristics and risk factors for mortality in Morganella morganii bacteremia. J Microbiol Immunol Infect 39: 328-334, 2006.

10. Kasper DL. Intraabdominal infections and abscesses. In: Harrison's Principles of Internal Medicine. 16th ed. Kasper DL, Braunwald E, Fauci AS, Hauser SL, Longo DL, Jameson JL, Eds. W.B. McGraw-Hill, New York, 2005: 749-754.
11. Coelho RF, Schneider-Monteiro ED, Mesquita JLB, Mazzucchi E, Marmo Lucon A, Srougi M. Renal and perinephric abscesses: analysis of 65 consecutive cases. World J Surg 31: 431-436, 2007.

12. Saint S, Lipsky BA. Preventing catheter-related bacteriuria: Should we? Can we? How? Arch Intern Med 159: 800-808, 1999.

13. O'Hara CM, Brenner FW, Miller JM. Classification, identification, and clinical significance of Proteus, Providencia, and Morganella. Clin Microbiol Rev 13: 534-546, 2000.

14. Tucci V, Isenberg HD. Hospital cluster epidemic with Morganella morganii. J Clin Microbiol 14: 563-566, 1981.

15. McDermott C, Mylotte JM. Morganella morganii: epidemiology of bacteremic disease. Infect Control 5: 131-137, 1984.

16. Heumann D, Roger T. Initial responses to endotoxins and Gramnegative bacteria. Clin Chim Acta 323: 59-72, 2002.

17. Van Amersfoort ES, Van Berkel TJ, Kuiper J. Receptors, mediators, and mechanisms involved in bacterial sepsis and septic shock. Clin Microbiol Rev 16: 379-414, 2003.

18. Tavio MM, Vila J, Ruiz J, Ruiz J, Martin-Sanchez AM, Jimenez de Anta MT. Resolution of high-molecular-weight components in lipopolysaccharides of Escherichia coli, Morganella morganii, Citrobacter freundii and Citrobacter diversus strains with sodium dodecyl sulfate polyacrylamide gels. J Microbiol Methods 39: $145-148,2000$

(C) 2008 The Japanese Society of Internal Medicine http://www.naika.or.jp/imindex.html 